\title{
Puffy hand syndrome
}

\section{The swelling is asymmetric and intermittent at first,} but over time becomes persistent and symmetric

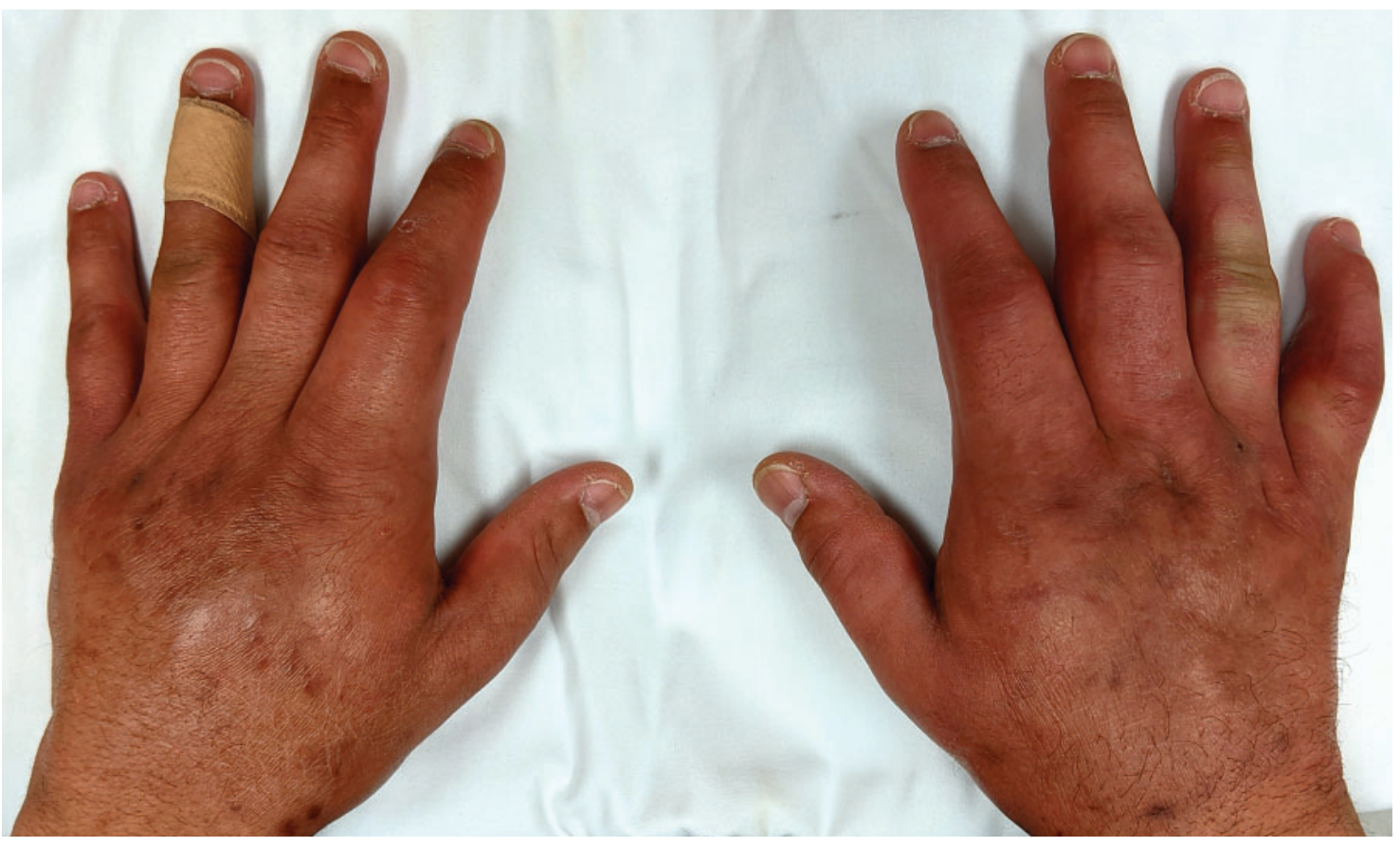

Figure 1. Both hands, including fingers and wrists, were diffusely swollen, with limited range of motion.

A 37-YEAR-OLD MAN presented to the rheumatology clinic for evaluation of swelling of his fingers and hands. The symptoms began 8 months earlier and at first were intermittent, but in the last 4 months, they had become persistent. The swelling started in the right (nondominant) hand, and then affected the left hand within a few weeks.

He reported limited range of motion in his fingers and wrists, with loss of hand dexterity and difficulty picking up objects or putting on socks and shoes. He denied any features suggestive of systemic sclerosis, including Raynaud phenomenon, ischemic digital ulcers, heartburn, dysphagia, dry cough, and exertional shortness of breath. He reported no doi:10.3949/ccjm.88a.20131 other chronic medical illness.

He said he smoked marijuana and half a pack of cigarettes per day. After 2 years of sobriety, he had restarted using intravenous (IV) drugs (opiates and methamphetamine) about 1 month earlier.

On examination, the dorsal and palmar aspects of both hands, fingers, and wrists were diffusely swollen with limited range of motion (Figure 1). However, there was no clinical evidence of inflammation (synovitis) of the wrist and finger joints, ie, no joint swelling, tenderness, heat, or redness.

Laboratory studies revealed a normal complete blood cell count, comprehensive metabolic panel, C-reactive protein, and thyroid-stimulating hormone level. Other tests with negative results included urine toxi- 
cology screen, hepatitis B and C and human immunodeficiency virus serology, and testing for rheumatoid factor, anti-cyclic citrullinated peptide antibody, antinuclear antibody, and antibodies to extractable nuclear antigens.

A radiograph of the right hand showed diffuse soft-tissue swelling (Figure 2). Computed tomography of the right hand also showed diffuse soft-tissue edema and skin thickening, most prominent over the dorsal side. There was no enhancing fluid collection or gas in the soft tissues and no bony cortical destruction. Based on the presentation, the history, and the evaluation, the diagnosis was puffy hand syndrome.

\section{A COMPLICATION OF IV DRUG USE}

Puffy hand syndrome, a common complication of IV drug use, is not a well-recognized condition among general practitioners. However, addiction specialists, dermatologists, and vascular medicine specialists are familiar with this syndrome.

In patients who do not volunteer a history of IV drug use, a rheumatologist may also be consulted, as the differential diagnosis includes scleroderma, mixed connective tissue disease, and the syndrome of remitting seronegative symmetrical synovitis with pitting edema (RS3PE). ${ }^{1}$

\section{DIAGNOSTIC CLUES}

The precise pathogenesis of puffy hand syndrome remains unknown. Hand edema, which is initially pitting, becomes more indurated over time, as there is progressive fibrosis of the subcutaneous tissues. The swelling is asymmetric and intermittent at first, often affecting the nondominant hand; over time, it becomes persistent and symmetric, as in this patient. ${ }^{1}$

Repeated injection of agents that can induce vascular and dermal sclerosis results in an inflammatory reaction with granuloma formation, similar to a foreign-body granulomatous reaction. Over time, the dense dermal fibrosis obscures the superficial veins and the extensor tendons on the dorsum of the hands. ${ }^{2}$ The edema is painless and nonpitting ${ }^{1}$ and is unaffected by elevation. ${ }^{2}$ Edema may also affect the feet when injection drug users use veins in the feet, legs, or groin.

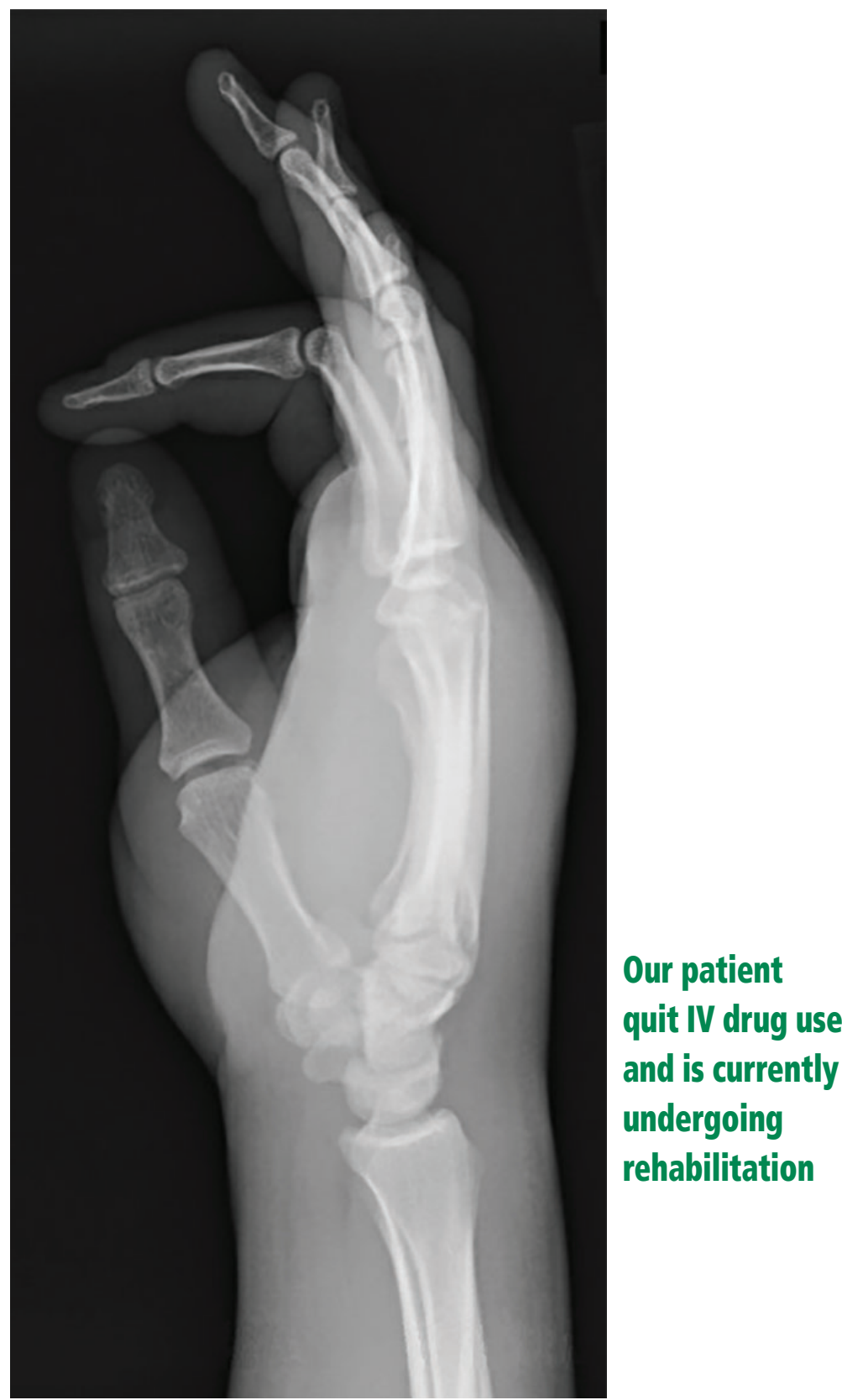

Figure 2. Radiography of the right hand showed diffuse soft-tissue swelling.

\section{Risk factors and underlying processes}

The main risk factors are repeated injections in the superficial veins on the dorsum of the hands (preferred by IV drug users because of easy access), and not using a tourniquet during the injections. ${ }^{3}$ The condition is more common in females. The risk increases with unsterile injection practices, suggesting that repeated local infections also contribute to 
the lymphatic destruction and consequent lymphedema. ${ }^{3}$

Neviaser et $\mathrm{al}^{2}$ performed venography, lymphangiography, and deep-skin biopsies in 4 patients with puffy hand syndrome. Deep venous patterns were entirely normal. In contrast, lymphangiograms showed extensive collateralization at the elbow, suggesting the destruction of deep lymphatic channels. Biopsy studies showed extensive fibrosis in the subcutaneous tissues. ${ }^{2}$

This study indicated that puffy hand syndrome results primarily from lymphatic obstruction, and that there may be concomitant local scarring resulting from inflammatory reactions at injection sites. ${ }^{2}$ Venous obstruction does not appear to play a primary role but can coexist in some cases. Repeated insults are needed for swelling to develop.

\section{A BROAD DIFFERENTIAL DIAGNOSIS}

The differential diagnosis of puffy hand syndrome is broad and includes conditions that cause anasarca, such as congestive heart failure, nephrotic syndrome, cirrhosis of the liver, and severe hypoalbuminemia. Other causes include upper-extremity venous thrombosis, deep palmar space infection, complex regional pain syndrome, lymphedema resulting after axillary lymph node removal or irradiation,

\section{REFERENCES}

1. Chouk M, Vidon C, Deveza E, et al. "Puffy hand syndrome." Joint Bone Spine 2017; 84(1):83-85. doi:10.1016/j.jbspin.2016.05.001

2. Neviaser RJ, Butterfield WC, Wieche DR. The puffy hand of drug addiction: a study of the pathogenesis. J Bone Joint Surg Am 1972; 54(3):629-633. pmid:5055159

3. Andresz V, Marcantoni N, Binder F, et al. Puffy hand syndrome due to drug addiction: a case-control study and filariasis.

Rheumatologic diseases causing puffy hands include rheumatoid arthritis, crystal arthropathies, systemic sclerosis, mixed connective tissue disease, nephrogenic systemic fibrosis, and RS3PE syndrome.

A thorough history and physical examination, followed by judicious use of laboratory and other investigations, should help determine the underlying cause of puffy hands.

\section{TREATMENT OPTIONS}

Treatment is mostly symptomatic. Intravenous drug use should stop permanently. Longterm use of low-stretch bandages and elastic compression gloves may be useful in decreasing the puffiness of the hands and fingers. ${ }^{4}$

\section{PATIENT OUTCOME}

Our patient quit IV drug use and is currently undergoing drug rehabilitation and psychiatric counseling. So far, he has not regained much movement in his hands and fingers.

Acknowledgment: The author thanks the patient for providing permission to share his information.

\section{DISCLOSURES}

The author reports no relevant financial relationships which, in the context of his contributions, could be perceived as a potential conflict of interest.

of the pathogenesis. Addiction 2006; 101(9):1347-1351. doi:10.1111/j.1360-0443.2006.01521.x

4. Arrault $\mathbf{M}$, Vignes $\mathbf{S}$. Puffy hand syndrome in drug addiction treated by low-stretch bandages. Ann Dermatol Venereol 2006; 133(10):769-772. [French.] doi:10.1016/s0151-9638(06)71040-7

Address: Soumya Chatterjee, MD, MS, FRCP, Department of Rheumatologic and Immunologic Disease, A50, Cleveland Clinic, 9500 Euclid Avenue, Cleveland, $\mathrm{OH} 44195$;

CHATTES@ccf.org 